\title{
THE BEST CONSTANT FOR THE CENTERED MAXIMAL OPERATOR ON RADIAL DECREASING FUNCTIONS
}

\section{J. M. Aldaz AND J. PÉREZ LÁZARo}

Abstract. We show that the lowest constant appearing in the weak type $(1,1)$ inequality satisfied by the centered Hardy-Littlewood maximal operator on radial, radially decreasing integrable functions is 1 .

Mathematics subject classification (2010): 42B25.

Keywords and phrases: Hardy-Littlewood maximal operator, radial functions,.

\section{REFERENCES}

[1] J. M. Aldaz, A general covering lemma for the real line. Real Analysis Exchange, 17 (1991-92), 394-398.

[2] J. M. AldaZ, Remarks on the Hardy-Littlewood maximal function. Proceedings of the Royal Society of Edinburgh A, 128 (1998), 1-9.

[3] J. M. AldaZ, A remark on the centered $n$-dimensional Hardy-Littlewood maximal function. Czechoslovak Math. J. 50 (125) (2000), no. 1, 103-112.

[4] J. M. ALDAZ, Dimension dependency of the weak type $(1,1)$ bounds for maximal functions associated to finite radial measures. Bull. Lond. Math. Soc. 39 (2007) 203-208. Also available at the Mathematics ArXiv.

[5] J. M. AlDAZ, The weak type $(1,1)$ bounds for the maximal function associated to cubes grow to infinity with the dimension. Available at the Mathematics ArXiv.

[6] J. M. Aldaz, L. Colzani, J. PÉrez LÁzaro, Optimal bounds on the modulus of continuity of the uncentered Hardy-Littlewood maximal function. Preprint.

[7] J. M. AldaZ, J. PÉrez LÁZARo, Functions of bounded variation, the derivative of the one dimensional maximal function, and applications to inequalities, Trans. Amer. Math. Soc., 359, 5 (2007), 2443-2461. Also available at the Mathematics ArXiv.

[8] J. M. AldAZ, J. PÉREZ LÁZARO, Boundedness and unboundedness results for some maximal operators on functions of bounded variation, J. Math. An. Appl., 337, 1 (2008), 130-143. Also available at the Mathematics ArXiv.

[9] J. M. Aldaz, J. Pérez LÁzaro, Behavior of weak type bounds for high dimensional maximal operators defined by certain radial measures, Positivity (to appear). Available at the Mathematics ArXiv.

[10] G. Aubrun, Maximal inequality for high-dimensional cubes, Confluentes Mathematici, 1, 2 (2009), 169-179, DOI No: 10.1142/S1793744209000067. Also available at the Mathematics ArXiv.

[11] J. M. AldaZ, J. L. VArona, Singular measures and convolution operators, Acta Math. Sin. (Engl. Ser.), 23, 3 (2007), 487-490.

[12] J. Bourgain, On high-dimensional maximal functions associated to convex bodies, Amer. J. Math., 108, 6 (1986), 1467-1476.

[13] J. Bourgain, On the $L^{p}$-bounds for maximal functions associated to convex bodies in $R^{n}$, Israel J. Math., 54, 3 (1986), 257-265.

[14] J. Bourgain, On dimension free maximal inequalities for convex symmetric bodies in $R^{n}$, Geometrical aspects of functional analysis (1985/86), 168-176, Lecture Notes in Math., 1267, Springer, Berlin, 1987. 
[15] A. CARBERY, An almost-orthogonality principle with applications to maximal functions associated to convex bodies, Bull. Amer. Math. Soc. (N.S.), 14, 2 (1986), 269-273.

[16] O. N. CAPRI, N. A. FAVA, Strong differentiability with respect to product measures, Studia Math. (1984), 173-178.

[17] L. Colzani, E. Laeng, C. Morpurgo, Symmetrization and norm of the Hardy-Littlewood maximal operator on Lorentz and Marcinkiewicz spaces, J. London Math. Soc., 77 (2008), 349-362.

[18] L. GRAFAKos, J. KinNUNEN, Sharp inequalities for maximal functions associated with general measures, Proc. Roy. Soc. Edinburgh Sect. A, 128, 4 (1998), 717-723.

[19] L. Grafakos, S. Montgomery-Smith, Best constants for uncentred maximal functions, Bull. London Math. Soc., 29, 1 (1997), 60-64.

[20] L. Grafakos, S. Montgomery-Smith, O. Motrunich, A sharp estimate for the HardyLittlewood maximal function. Studia Math., 134, 1 (1999), 57-67.

[21] J. Kinnunen, The Hardy-Littlewood maximal function of a Sobolev function, Israel J. Math., 100 (1997), 117-124.

[22] A. D. Melas, On the centered Hardy-Littlewood maximal operator, Trans. Amer. Math. Soc., 354, 8 (2002), 3263-3273.

[23] A. D. Melas, The best constant for the centered Hardy-Littlewood maximal inequality, Ann. of Math.(2), 157, 2 (2003), 647-688.

[24] M. T. Menárguez, F. Soria, On the maximal operator associated to a convex body in $R^{n}$, Collect. Math., 43, 3 (1992), 243-251 (1993).

[25] D. MÜLLER, A geometric bound for maximal functions associated to convex bodies, Pacific J. Math., 142, 2 (1990), 297-312.

[26] E. M. StEIn, The development of square functions in the work of A. Zygmund, Bull. Amer. Math. Soc. (N.S.), 7, 2 (1982), 359-376.

[27] E. M. STEIN, Three variations on the theme of maximal functions, Recent progress in Fourier analysis (El Escorial, 1983), 229-244, North-Holland Math. Stud., 111, North-Holland, Amsterdam, 1985.

[28] E. M. STEIN, Harmonic analysis: real-variable methods, orthogonality, and oscillatory integrals, with the assistance of Timothy S. Murphy, Princeton Mathematical Series, 43. Monographs in Harmonic Analysis, III. Princeton University Press, Princeton, NJ, 1993.

[29] E. M. Stein, J. O. Strömberg, Behavior of maximal functions in $R^{n}$ for large $n$, Ark. Mat., 21, 2 (1983), 259-269. 